\title{
Actividad física, estrés y su relación con el indice de masa corporal en docentes universitarios en pandemia
}

\section{Physical activity, stress and its relationship with the body mass index in pandemic university teachers}

\author{
a alcidesflores@unap.edu.pe \\ b danielo c p@outlook.com \\ calbertosantoss615@gmail.com \\ dcyapuchura@unap.edu.pe \\ eympino@unap.edu.pe
}

Alcides Flores Paredes a 0000-0001-6355-413X

Daniel Coila Pancca ${ }^{\mathrm{b}}$ 0000-0003-3756-8401

Santos Alberto Ccopa ${ }^{c}$ 0000-0001-6906-8079

Cristobal Rufino Yapuchura Saico ${ }^{\mathrm{d}}$ 0000-0003-1956-3922

Yony Martín Pino Vanegas e 0000-0002-5414-6262

Universidad Nacional del Altiplano, Puno, Perú.

\author{
Recibido: 06/05/2021 \\ Aprobado: 23/08/2021 \\ Publicado: 15/09/2021
}

\section{Resumen}

La actividad física realizada de manera sistemática es la piedra angular frente a las enfermedades. Los docentes universitarios por las múltiples responsabilidades, y viviendo en confinamiento son propensos a desarrollar el estrés y el sobrepeso. El objetivo de la investigación es determinar la relación de la actividad física, el estrés con el Índice de Masa Corporal en docentes universitarios en pandemia. El tipo de investigación es básica, con diseño descriptivo correlacional múltiple con una población de 1150 docentes y con una muestra de tipo probabilística de 234 docentes (150 varones y 84 mujeres) con promedio de edad $49.70 \pm 10.01$, los instrumentos utilizados son los cuestionarios de actividad física, estrés y el reporte de sus medidas antropométricas (estatura y peso). Resultados el $58.86 \%$ de varones presentan actividad física baja, en comparación con las mujeres $41.14 \%$, el 91.95\% muestran estrés por presión laboral, los varones presentan mayor tendencia al sobrepeso $67.59 \%$, en comparación con las mujeres $32.41 \%$ y obesidad grado II $33.96 \%$. Se determina la relación entre las variables actividad física, estrés y el Índice de Masa Corporal con un Rho de Spearman inversamente proporcional de -0,183 y -0,204. En conclusión, los docentes varones muestran porcentajes más altos de inactividad física y sobrepeso.

\begin{abstract}
Systematic physical activity is the cornerstone against disease. University teachers due to multiple responsibilities and living in confinement are prone to developing stress and being overweight. The objective of the research is to determine the relationship between physical activity, stress and bodymass indexinuniversity teachersinapandemic. The type of research is basic, with a multiple correlational descriptive design with a population of 1150 teachers and a probabilistic sample of 234 teachers (150 men and 84 women) with an average age of $49.70 \pm 10.01$, the instruments used are questionnaires of physical activity, stress and the report of their anthropometric measurements (height and weight). Results $58.86 \%$ of men present low physical activity, compared to women $41.14 \%, 91.95 \%$ show stress due to work pressure, men have a greater tendency to be overweight $67.59 \%$, compared to women $32.41 \%$ and obesity grade II $33.96 \%$. The relationship between the variables physical activity, stress and body mass index is determined with an inversely proportional Spearman Rho of -0.183 and -0.204. In conclusion, male teachers show higher percentages of physical inactivity and overweight.
\end{abstract}

Keywords: Physical activity; stress; pandemic; overweight obesity; healthy life.

Palabras clave: Actividad física; estrés; pandemia; sobrepeso obesidad; vida saludable. 


\section{Introducción}

Los docentes universitarios por su recargada labor académica antes de la pandemia mostraban una alta frecuencia de sobrepeso y obesidad, además alto riesgo cardiovascular y metabólico (Morales et al., 2018). Y el contexto global en el que vivimos producto de la emergencia sanitaria, trajo un confinamiento y aislamiento en las casas y por consiguiente un sedentarismo en los docentes universitarios. En España, un tercio de los menores $\mathrm{y}$ dos tercios de los adultos padecen exceso de peso, una condición que genera un sobrecoste médico directo de 2000 millones de euros y el entorno alimentario obesogénico causa obesidad al promover el consumo de bebidas azucaradas y de alimentos ultraprocesados (Royo et al., 2019).

La pandemia trajo una serie de emociones, sentimientos y percepciones al docente con nuevos desafíos en sus diferentes actividades. Sin embargo, es importante fomentar el establecimiento de procesos de reflexión en torno al equilibrio físico y mental dentro y fuera del entorno universitario (Santos et al., 2021).

Córdova (2016) señala que la obesidad y las enfermedades crónicas no trasmisibles representan la verdadera pandemia mundial en salud. En la misma línea, Arrieta y Pedro-Botet (2020) refieren que es medular tener en cuenta que, en nuestro medio, la obesidad es en gran medida producto de un ambiente obesogénico, consistente en dietas hipercalóricas promovidas mayoritariamente por fuentes comerciales, acompañado de una actividad física mínima en el contexto de una baja cultura alimenticia e importantes lagunas en dietética.

El índice abdominal visceral (VAI) es un método útil para definir a aquellos sujetos con adiposidad visceral en nuestra región. La edad, el consumo calórico diario y la glucemia alterada en ayuno son los principales factores asociados con los valores más elevados del índice, mientras que la actividad física durante el ocio es un factor protector para clasificar a las personas en los estadios más avanzados (Ortiz et al., 2017). Las personas con síndrome metabólico y sobrepeso obesidad que afirman cumplir las recomendaciones sobre Actividad Física (AF) alcanzan mayor VO2máx además las actividades sedentarias no influyen en el VO2máx (Tojal et al., 2020).
Russo et al. (2020) sugieren que el ejercicio físico se utiliza como estrategia ampliamente disponible para fortalecer el funcionamiento cognitivo, especialmente de las funciones ejecutivas $y$ memoria, y retardar la aparición de la demencia. Sin embargo, es importante considerar que las desigualdades socioeconómicas en las prácticas de actividad física deben ser consideradas en las intervenciones de salud pública (Brazo et al., 2018).

Ramírezetal.(2012) sostienen que el entrenamiento de intensidad vigorosa se relaciona con menores valores en los marcadores de sobrepeso y obesidad en mujeres, pero no en hombres. Igualmente Escobar y Divisón (2016) afirman que el ejercicio físico se asocia con un descenso de la mortalidad total, parece que existe una curva en J, de tal forma quemientras que realizarjogging con unaintensidad leve o moderada se asociaba con un descenso de la mortalidad. Asimismo, aplicar intervenciones de actividad física de intensidad suave es una garantía de mejora en la salud funcional y la calidad de vida en las personas mayores (Font et al., 2020).

Realizar sólo 30 minutos de actividad física a la semana reduce el riesgo de Diabetes Mellitus Tipo 2 (DMT2) en un 7\%, los beneficios de realizar una mayor cantidad de actividad física podrían llegar a reducir en $47 \%$ el riesgo de desarrollarla. Las implicaciones de estos resultados, en términos de salud pública, son muy relevantes, considerando que un modesto aumento en los niveles de actividad física de la población podría reducir el riesgo de esta patología, como también así la carga económica, al disminuir las complicaciones asociadas a la calidad de vida de las personas que padecen esta enfermedad (Salas et al., 2018).

Está claro que tanto la prevención como el tratamiento de la obesidad requieren un enfoque multifacético que se centra, en gran medida, en el estilo de vida. Dentro del estilo de vida juega un papel fundamental la dieta y su combinación con el ejercicio y terapia cognitivo conductual es la forma más eficiente de intervención en el sobrepeso y la obesidad (Gómez y Martínez-Marcos, 2018; Naranjo et al., 2021).

Los cambios hacia un estilo de vida cardiosaludable en el hombre adulto, deberían comenzar por el control médico preventivo a partir de los 30 años de edad y considerar en general, actividad física regular, nutrición cardiosaludable, suspensión 
completa del tabaquismo y control del peso corporal (Rondanelli y Rondanelli, 2014). Volviendo al tema que nos ocupa un programa de AF y motivación incrementa la AF y la autoestima en adultos usuarios de atención primaria, además resulta tener más seguimiento y aumenta la autoestima mejora el control de la presión arterial sistólica (PAS) en hipertensos y por tanto mejora los parámetros antropométricos y lipídicos (López et al., 2020; Villalobos et al., 2019).

Dicho lo anterior un programa de ejercicio físico y de entrenamiento concurrente mejora significativamente la composición corporal en personas obesas, que están relacionadas con los niveles de fuerza muscular mejorada y conduce a una disminución significativa de la grasa visceral y puede ser una herramienta complementaria útil para la prevención, optimizar la recuperación, mejorar la calidad de vida y brindar protección inmunológica contra el SARS-CoV-2 Infección por virus a largo plazo (Fernández et al., 2020; Simón et al., 2020).

Por otro lado, el programa de técnicas tales como entrenamiento en relajación, reestructuración cognitiva y manejo del tiempo resulta efectivo para disminuir el nivel de estrés y ansiedad en los participantes, ayudando a mejorar la calidad de vida de los estudiantes y los profesionales docentes y no docentes que participaron (Iglesias et al., 2018).

Las múltiples responsabilidades académicas de investigación, responsabilidad social, preparación de clases, tutoría y acreditación genera inactividad física, estrés y por consiguiente sobrepeso, obesidad y si no se asumen estilos de vida saludable de manera responsable serán más propensos a las enfermedades crónico no transmisibles (ECNT). En esa línea el objetivo fue determinar la relación de la actividad física, estrés con el índice de masa corporal - IMC en docentes universitarios en pandemia, analizando los niveles de actividad física, el estrés y sus dimensiones que presentan asociados al sobrepeso y la obesidad de los catedráticos en esta emergencia sanitaria.

\section{Marco Teórico}

\section{Actividad física}

La Organización Mundial de la Salud (2020) "sostiene que la actividad física es cualquier movimiento corporal realizado por los músculos esqueléticos, con el propósito de consumir de energía calórica. Esto comprende las actividades realizadas de trabajar, jugar y viajar, las tareas domésticas y las actividades recreativas" (p.1).

Por otra parte según la OMS (2010) plantea la importancia de realizar actividad física moderada e intensa y que los adultos de 18 a 64 años deben cumplir las siguientes actividades físicas de:

- Lograr un mínimo de 150 minutos semanales de actividad física aeróbica moderada, o bien un mínimo de 75 minutos semanales de actividad aeróbica vigorosa, o bien una combinación equivalente de actividad moderada y vigorosa.

- Los adultos para conseguir mayores beneficios deberían incrementar esos niveles hasta 300 minutos semanales de actividad aeróbica moderada, o bien 150 minutos de actividad aeróbica vigorosa cada semana; acompañado del fortalecimiento muscular de los grandes grupos musculares dos o más días a la semana.

El ejercicio físico contribuye a mejorar el estado físico y mental de las personas como factor de protección, promoción y mantenimiento de la salud, el bienestar y la calidad de vida de los mayores ayudando a reducir su estrés y mejorar la cognición, capacidad de pensamiento, y fortalecer las habilidades funcionales (Limón y Ortega, 2011). Del mismo modo, García y Froment (2018) resaltan cómo la actividad física afecta a la calidad de vida de los mayores en tres aspectos psicológicos principales: distracción, autoeficacia y relaciones sociales.

La práctica de actividad física supone una distracción en la cotidianidad y fortalece el estado de ánimo, la motivación para realizar actividad física supone a su vez un factor esencial para el incremento del autoconcepto, la autodeterminación y la autoeficacia. Por último, la práctica de actividad física permite la interacción social y la cooperación con otros (Martínez et al., 2020).

\section{El estrés}

El estrés es un concepto principal para comprender tanto la vida como la evolución. El futuro de los seres humanos como especie depende en gran parte de la capacidad para adaptarse a factores estresantes potentes. Todos los seres humanos 
están expuestos a situaciones estresantes a nivel social, comunitario e interpersonal, donde la manera en que se enfrentan estos desafíos definirá en gran medida la salud de la sociedad. Las personas con respuestas de afrontamiento eficaces pueden beneficiarse de tales experiencias y lidiar bien con los factores estresantes crónicos (Iglesias et al., 2018).

\section{El sedentarismo}

El término sedentarismo proviene del vocablo en latín sedere, que significa sentado, e intuitivamente se suele asociar sedentarismo a falta de actividad física; de hecho, muchos estudios se refieren a participantes sedentarios cuando estos no alcanzan un determinado nivel de actividad física, además es, entendido como la ausencia de actividad física o tendencia a la falta de movimiento (Liévano et al., 2015).

\section{El índice de masa corporal (IMC)}

El IMC o índice de Quetelet se define como el peso $(\mathrm{Kg}) /$ cuadrado de la estatura en $\mathrm{m}^{2}$. El IMC tiene una alta correlación con el peso y es independiente de la estatura. Esta propiedad presenta al IMC como un buen índice para caracterizar el comportamiento del peso en correspondencia o en relación con la estatura del individuo, caracterizando de esta forma las dimensiones corporales de cada sujeto (Monterrey y Porrata, 2001). La OMS (2020) indica que la prueba para determinar si una persona ${ }_{78}$ se encuentra en sobrepeso u obesidad es el IMC a partir de la toma de medidas bioantropométricas de peso corporal y estatura al cuadrado.

Tabla 1. Valores límites del IMC

\begin{tabular}{lll}
\hline Clasificación & IMC(Kg/m $\left.\mathbf{m}^{2}\right)$ & $\begin{array}{l}\text { Riesgo } \\
\text { asociado a la } \\
\text { salud }\end{array}$ \\
\hline Normo peso & $18,5-24,9$ & Promedio \\
\hline Exceso de peso & $\geq 25$ & Aumentado \\
\hline $\begin{array}{l}\text { Sobrepeso o pre } \\
\text { obeso }\end{array}$ & $25-29,9$ & Aumento \\
\hline $\begin{array}{l}\text { Obesidad grado I } \\
\text { o moderado }\end{array}$ & $30-34,9$ & $\begin{array}{l}\text { Aumento } \\
\text { severo }\end{array}$ \\
\hline $\begin{array}{l}\text { Obesidad grado } \\
\text { II o severa }\end{array}$ & $35-39,9$ & \\
\hline
\end{tabular}

\section{Salud}

Tejada et al. (2016) consideran que la salud es algo más que la ausencia de la enfermedad, se trata de una realidad que se va edificando en un proceso dinámico, que se desarrolla y potencia continuamente. Por otra parte, en 1984 la OMS la define como "el estado de completo bienestar, mental y social".

\section{Metodología}

Se propuso el enfoque cuantitativo, el tipo de investigación básica y diseño descriptivo correlacional múltiple Hernández et al. (2006) la población fue de 1150 docentes de la Universidad Nacional del Altiplano ubicado en la región de Puno. La muestra es de tipo probabilística aleatoria simple de 234 docentes (150 varones con promedio de edad $52.18 \pm$ (DS) 9.12 y 84 mujeres con 45.19 \pm (DS) 8.51 .

Los instrumentos utilizados fueron el cuestionario internacional de actividad física para uso con jóvenes y adultos de mediana edad (15-69 años) IPAQ (Di Blasio et al., 2017), el cuestionario de estrés de Alvitesí (2019) donde se contextualizó algunos ítems además se tuvo un análisis de confiabilidad estadística, para lo cual se aplicó la prueba piloto con el apoyo de Google drive y se obtuvo un Alfa de Cronbach de 0,948 y la aplicación de las mediciones antropométricas (estatura y peso) (Stewart et al., 2011).

\section{Procedimiento}

La recolección de datos se realizó a través de una encuesta autoadministrada en línea por Google drive para evaluar la actividad física, el estrés, y la auto medición antropométrica del peso corporal y la estatura. Los protocolos de investigación se probaron previamente en un estudio piloto, realizándose la validez respectiva por un docente investigador experto en el área, para la medición del peso corporal se consideró la menor cantidad de ropa (polo, short y descalzo) para la medición de la estatura reportaron su medición en centímetros y descalzo.

Dichos cuestionarios en el primer apartado tenían el consentimiento informado si deseaban ser partícipes continuaban con el llenado de los cuestionarios y la otra opción de no participar si elegían la segunda opción se terminaba el 
cuestionario. Respecto a los aspectos bioéticos, el presente estudio guarda la confidencialidad de los datos, además se tuvo una lista anónima para realizar el análisis estadístico.

Para el proceso estadístico se utilizó el análisis descriptivo de frecuencias, porcentajes, medidas de tendencia central y la estadística inferencial procesados con el programa SPSS Statistics versión 26, determinándose el promedio, desviación estándar y el estadístico de prueba con un intervalo de confianza del $95 \%$.

\section{Resultados y discusión}

Tabla 2. Actividad física por sexo en docentes de la Universidad Nacional del Altiplano.

\begin{tabular}{llllllll}
\hline \multirow{2}{*}{$\begin{array}{l}\text { Niveles de la } \\
\text { actividad física }\end{array}$} & \multicolumn{3}{l}{ Mujer } & \multicolumn{2}{l}{ Varón } & \multicolumn{2}{c}{ Total } \\
\cline { 2 - 8 } Baja & f & $\%$ & f & $\%$ & f & $\%$ \\
\hline Moderada & 65 & 41.14 & 93 & 58.86 & 158 & 100 \\
\hline Alta & 14 & 25.45 & 41 & 74.55 & 55 & 100 \\
\hline Total & 5 & 23.81 & 16 & 76.19 & 21 & 100 \\
\hline
\end{tabular}

De los 234 docentes universitarios $58.86 \%$ de varones se ubican en la escala de nivel de actividad baja, $74.55 \%$ en la escala moderada y $76.19 \%$ en la escala alta, en diferencia con las mujeres $41.14 \%$ presentan actividad física baja, $25.45 \%$ se ubican en la escala moderada, y $23.81 \%$ se sitúan en la escala alta.

Los varones presentan mayor actividad física moderada y alta frente a las mujeres, resultados diferentes encontrados con Aparicio et al. (2016) donde las mujeres presentaban mayor grado de Actividad Física (AF) (en especial si se tiene en cuenta la AF doméstica), y esto podría explicar en parte la mayor proporción de OMS observada en ellas. De igual modo, el mayor grado de AF observado en las mujeres también podría explicar en parte el perfil cardiometabólico más favorable observado en ellas; sin embargo, se requiere asumir la práctica de actividad física de manera sistemática y planificada. Igualmente Aljuhani et al. (2020) mostró que los niveles bajos de actividad física sedentaria se asociaron significativamente con el riesgo de tener síndrome metabólico en los hombres, hallazgos similares con el estudio donde el $58.86 \%$ de varones presentan actividad física baja, en la emergencia sanitaria.

Coincidimos con Dong et al. (2021) donde demuestra que la AF regular puede reducir de forma independientemente el nivel de ácido úrico en suero y la prevalencia de la Hiperuricemia (HUA), pero el Tiempo Sentado (ST) prolongado puede aumentar significativamente el nivel de ácido úrico en suero y HUA. Además, niveles excesivos de ST ( $>6 \mathrm{~h} /$ día) podrían contrarrestar el efecto beneficioso de la AF en HUA. Estos hallazgos sugieren que tanto la $\mathrm{AF}$ regular como la reducción de (ST) son importantes para prevenir el HUA. Además, Las intervenciones centradas en la reducción de (TS) excesiva pueden ser una estrategia más eficaz para disminuir el riesgo de HUA, que debe recibir más atención en la salud pública.

Hernández et al. (2018) muestran que los pacientes en hemodiálisis estudiados tienen un peso adecuado para la talla, pero con menos masa muscular y fuerza muscular disminuida para la edad; el nivel de actividad física fue sedentario y la calidad de vida evaluada mostró una menor puntuación en el componente físico.

Tabla 3. Actividad física y su relación con el estrés en docentes de la Universidad Nacional del Altiplano.

\begin{tabular}{|c|c|c|c|c|c|c|c|c|c|c|c|c|}
\hline \multirow[t]{2}{*}{$\begin{array}{l}\text { Actividad } \\
\text { Física }\end{array}$} & \multicolumn{2}{|c|}{$\begin{array}{l}\text { Estrés por mal } \\
\text { afrontamiento }\end{array}$} & \multicolumn{2}{|c|}{$\begin{array}{l}\text { Estrés por } \\
\text { presión } \\
\text { laboral }\end{array}$} & \multicolumn{2}{|c|}{$\begin{array}{l}\text { Estrés por } \\
\text { creencias } \\
\text { desadaptativas }\end{array}$} & \multicolumn{2}{|c|}{$\begin{array}{l}\text { Estrés por } \\
\text { depresión }\end{array}$} & \multicolumn{2}{|c|}{$\begin{array}{l}\text { Estrés por } \\
\text { ansiedad }\end{array}$} & \multicolumn{2}{|c|}{ Total } \\
\hline & $f$ & $\%$ & $\mathrm{f}$ & $\%$ & $\mathrm{f}$ & $\%$ & $\mathrm{f}$ & $\%$ & $\mathrm{f}$ & $\%$ & $\mathrm{f}$ & $\%$ \\
\hline Baja & 4 & 10.26 & 80 & 91.95 & 15 & 100 & 39 & 81.25 & 20 & 44.44 & 158 & 67.52 \\
\hline Moderada & 20 & 51.28 & 1 & 1.15 & 0 & 0 & 9 & 18.75 & 25 & 55.56 & 55 & 23.50 \\
\hline Alta & 15 & 38.46 & 6 & 6.90 & 0 & 0 & 0 & 0 & 0 & 0.00 & 21 & 8.97 \\
\hline Total & 39 & 89.74 & 87 & 8.05 & 15 & 100 & 48 & 18.75 & 45 & 55.56 & 234 & 100 \\
\hline
\end{tabular}

Los catedráticos universitarios presentan mayor nivel de actividad física baja $67.52 \%$, y muestran estrés por presión laboral $91.95 \%$, estrés por depresión $81.25 \%$, estrés por ansiedad $44.44 \%$, estrés por creencias desadaptativas $100 \%$, y revelan estrés por mal afrontamiento $10.26 \%$, 
en el nivel de actividad moderada $23.50 \%$, y presentan estrés por ansiedad $55.56 \%$, estrés por mal afrontamiento $51.28 \%$, estrés por depresión $18.75 \%$, estrés por presión laboral $1.15 \%$ y no se encuentran resultados en estrés por creencias desadaptativas, y en el nivel de actividad física alta $8.97 \%$ y exhiben estrés por mal enfrentamiento $38.46 \%$, estrés por presión laboral $6.90 \%$ y no se encuentran resultados en las demás dimensiones.

Los docentes presentan mayor estrés por presión laboral 91.95\% datos similares encontrados con Alvitesí, (2019) donde existe correlación significativa entre el estrés docente y los factores psicosociales, $(\mathrm{p}=.000)$, prevaleciendo en su mayoría el nivel de estrés por ansiedad, depresión, creencias desadaptativas que se correlacionan con los factores psicosociales. Tacca y Tacca (2019) sustentan que los docentes contratados presentan mayor nivel de estrés y que los principales síntomas experimentados son el cansancio, no poder dormir, dolores de cabeza, poca concentración y variación del apetito.

Se considera ahora que existen diferencias significativas en función del sexo, el estado civil y el nivel de enseñanza de los profesores, resultando en mayores niveles de agotamiento para las profesoras de nivel escolar y niveles moderados de despersonalización para los profesores universitarios, en quienes se obtuvieron correlaciones negativas con el número de hijos y el síndrome de burnout (Arias et al., 2019).
Avanzando en nuestro razonamiento la presencia de estrés organizacional, incrementa la intensidad de violencia y la presencia de acoso psicológico (Acosta et al., 2019). Consideramos ahora el agotamiento emocional varía significativamente según área de desempeño de los profesores (Bedoya et al., 2017).

Mesurado y Laudadío (2019) revelan que los docentes más experimentados presentan mayores niveles de capital psicológico (específicamente eficacia, resiliencia y esperanza) y mayor nivel de absorción, una dimensión de engagement, que los menos experimentados. Se debe destacarse la actividad física como una posible alternativa para reducir la carga de la pandemia de COVID-19 sobre la función cognitiva y la salud mental (Feter et al., 2021).

Es necesario implementar medidas preventivas en las labores de los académicos para evitar que su calidad de vida y salud sufran un mayor deterioro (Palacios y Montes de Oca, 2017). Conjuntamente se requiere de una atención plena para la reducción del estrés en trabajadores universitarios (Oblitas et al., 2020). El ejercicio físico es piedra angular en el tratamiento de pacientes con sobrepeso/ obesidad, principalmente con efectos a nivel funcional, metabólico, muscular y cardiovascular. Con dosis adecuadas de ejercicio físico también se podrían favorecer principalmente cambios en la composición corporal del paciente (Burgos et al., 2017).

Tabla 4. Sexo y su relación con el IMC en docentes de la Universidad Nacional del Altiplano.

\begin{tabular}{|c|c|c|c|c|c|c|c|c|c|c|c|c|}
\hline \multirow[b]{2}{*}{ Sexo } & \multicolumn{2}{|c|}{ Normopeso } & \multicolumn{2}{|c|}{$\begin{array}{l}\text { Sobrepeso } \\
\text { (Obesidad grado } \\
\text { I) } 25-29,9\end{array}$} & \multicolumn{2}{|c|}{$\begin{array}{l}\text { Obesidad } \\
\text { grado II. } 30 \text { - } \\
\mathbf{3 4 , 9} \\
\end{array}$} & \multicolumn{2}{|c|}{$\begin{array}{l}\text { Obesidad } \\
\text { grado III. 35- } \\
39,9\end{array}$} & \multicolumn{2}{|c|}{$\begin{array}{l}\text { Obesidad } \\
\text { grado IV. }>40\end{array}$} & \multicolumn{2}{|c|}{ Total } \\
\hline & $\mathrm{f}$ & $\%$ & $\mathrm{f}$ & $\%$ & $\mathrm{f}$ & $\%$ & $\mathrm{f}$ & $\%$ & $\mathrm{f}$ & $\%$ & $\mathrm{f}$ & $\%$ \\
\hline Mujer & 25 & 37.88 & 35 & 32.41 & 18 & 33.96 & 5 & 83.33 & 1 & 100 & 84 & 35.90 \\
\hline Varón & 41 & 62.12 & 73 & 67.59 & 35 & 66.04 & 1 & 16.67 & 0 & 0 & 150 & 64.10 \\
\hline Total & 66 & 100 & 108 & 100 & 53 & 100 & 6 & 100 & 1 & 100 & 234 & 100 \\
\hline
\end{tabular}

Los docentes varones presentan mayor sobrepeso $y$ se ubican en la escala sobrepeso $67.59 \%$, normopeso $62.12 \%$, obesidad grado II $66.04 \%$, obesidad grado III $16,67 \%$, y no se encuentran datos en la escala obesidad grado IV, en contraste con las mujeres presentan sobrepeso $32.41 \%$, normopeso $37.88 \%$, obesidad grado II $33.96 \%$, obesidad grado III $83.33 \%$ y $100 \%$ en obesidad grado IV.
Los hallazgos del presente estudio coinciden con Flores et al., (2019) donde existe mayor prevalencia de sobrepeso y obesidad en docentes varones, en comparación con las mujeres; además Mejia et al. (2020) mencionan que existe una asociación $\mathrm{y}$ tendencia positiva entre el tiempo laboral y el incremento del diámetro de cintura, también se halló asociación entre el sexo masculino y del aumento del IMC de los trabajadores. Por último, ambas medidas antropométricas se incrementan con el pasar de los años. 
De manera semejante Enriquez et al. (2021) indican que las personas con normo y sobrepeso muestran mejores capacidades físicas, así como valores elevados de actividad física, mientras que altos índices de grasa corporal total y un IMC elevado son un factor determinante para el desarrollo de las mismas.

Respecto a lo anterior es pertinente asumir comida saludable Ortiz et al. (2019) muestran que la inclusión de hidratos de carbono de bajo índice glucémico (HC-BIG) en la dieta diaria parece influir en el aumento de las concentraciones de los triglicéridos (TG) al término de un ejercicio de intensidad moderada, indicando que la liberación lenta de glucosa al torrente sanguíneo genera una mayor utilización de lípidos durante el ejercicio; esto significaría una vía efectiva hacia el control del peso corporal adecuado.

Tabla 5. Actividad física y su relación con el IMC en docentes de la Universidad Nacional del Altiplano.

\begin{tabular}{|c|c|c|c|c|c|c|c|c|c|c|c|c|}
\hline \multirow[t]{2}{*}{$\begin{array}{l}\text { Actividad } \\
\text { Física }\end{array}$} & \multicolumn{2}{|c|}{ Normopeso } & \multicolumn{2}{|c|}{$\begin{array}{l}\text { Sobrepeso } \\
\text { (Obesidad } \\
\text { grado I) 25-29,9 }\end{array}$} & \multicolumn{2}{|c|}{$\begin{array}{l}\text { Obesidad } \\
\text { grado II. } \\
\text { 30-34,9 } \\
\end{array}$} & \multicolumn{2}{|c|}{$\begin{array}{l}\text { Obesidad } \\
\text { grado III. } \\
\mathbf{3 5 - 3 9 , 9} \\
\end{array}$} & \multicolumn{2}{|c|}{$\begin{array}{l}\text { Obesidad } \\
\text { grado IV. } \\
>40\end{array}$} & \multicolumn{2}{|c|}{ Total } \\
\hline & $f$ & $\%$ & $\mathrm{f}$ & $\%$ & $\mathrm{f}$ & $\%$ & $\mathrm{f}$ & $\%$ & $\mathrm{f}$ & $\%$ & $\mathrm{f}$ & $\%$ \\
\hline Baja & 41 & 62.12 & 66 & 61.11 & 51 & 96.23 & 0 & 0 & 0 & 0 & 158 & 67.52 \\
\hline Moderada & 5 & 7.58 & 42 & 38.89 & 2 & 3.77 & 6 & 100 & 0 & 0 & 55 & 23.50 \\
\hline Alta & 20 & 30.30 & 0 & 0.00 & 0 & 0.00 & 0 & 0 & 1 & 100 & 21 & 8.97 \\
\hline Total & 66 & 37.88 & 108 & 38.89 & 53 & 3.77 & 6 & 100 & 1 & 100 & 234 & 100.00 \\
\hline
\end{tabular}

Los docentes universitarios se ubican en el nivel de actividad física baja $67.52 \%$ y se ubican en sobrepeso con $61.11 \%$, en obesidad grado II $96.23 \%$, en normopeso $62.12 \%$ y no se encuentran resultados en las escalas de obesidad grado III y IV, en el nivel de actividad física moderada $23.50 \%$ y se sitúan en sobrepeso $38.89 \%$, obesidad grado III $100 \%$, normopeso $7.58 \%$, obesidad grado II $3.77 \%$ y no encontramos datos en la escala obesidad grado IV y en el nivel de actividad física alta $8.97 \%$, en normopeso $30.30 \%$, obesidad grado IV $100 \%$ y no hallamos resultados en las escalas obesidad grado I, II y III.

Los docentes presentan mayor sobrepeso (obesidad grado I) datos similares con Nepal et al. (2020) en sus hallazgos muestra que la prevalencia de sobrepeso y obesidad fue alta. Se debe seguir ejercicio físico regular y una dieta equilibrada para prevenir el sobrepeso y las enfermedades no transmisibles. Hsiao et al. (2020) argumentan que la participación de un programa integral para mujeres menopaúsicas proporciona experiencias ricas en estímulos positivos, la alegría de la sudoración deportiva, cocinar y experimentar la comida, un plan que comprende consejo, asistencia y seguimiento en la atención y eficacia.

La salud es lo más preciado que posee el ser humano se debe generar una cultura de prevención más sólida en la sociedad, que origine un verdadero cambio en los estilos de vida de los docentes (Córdova, 2016). Los menores tienden a replicar el estilo de vida (lo que incluye los hábitos de actividad física) de los adultos con los que conviven. Lo que convierte a los adultos en la población diana sobre la que incidir políticas sanitarias de prevención primaria y secundaria para mejorar su salud y la de los niños que los rodean (Brais et al., 2021).

Tabla 6. Correlación de Rho de Spearman entre las variables actividad física y el estrés en docentes de la Universidad Nacional del Altiplano.

\begin{tabular}{lllcc}
\hline & & \multicolumn{1}{c}{$\begin{array}{c}\text { Actividad } \\
\text { física }\end{array}$} & Estrés \\
\hline & & $\begin{array}{l}\text { Coeficiente de } \\
\text { Actividad } \\
\text { física }\end{array}$ & 1,000 &,$- 183^{* *}$ \\
\cline { 2 - 5 } correlación & Sig. (bilateral) &. &, 005 \\
\cline { 2 - 5 } $\begin{array}{l}\text { Rho de } \\
\text { Spearman }\end{array}$ & $\begin{array}{l}\text { Coeficiente de } \\
\text { correlación }\end{array}$ &,$- 183^{* *}$ & 1,000 \\
\cline { 2 - 5 } & Estrés & Sig. (bilateral) &, 005 &. \\
\cline { 2 - 5 } & $\mathrm{N}$ & 234 & 234 \\
\hline
\end{tabular}

**. La correlación es significativa en el nivel 0,01 (bilateral).

Según la correlación Rho de Spearman entre las variables actividad física y el estrés es negativa muy baja de $-0,183$ con un nivel de significancia de $\mathrm{P}=0,005<0,01$; por lo tanto, se establece una correlación inversamente proporcional entre las variables actividad física y el estrés. Razón por la cual a mayor actividad física es menor el estrés en los docentes universitarios. 
Tabla 7. Correlación de Rho de Spearman entre las variables actividad física y el IMC en docentes de la Universidad Nacional del Altiplano.

\begin{tabular}{|c|c|c|c|c|}
\hline & & & $\begin{array}{l}\text { Actividad } \\
\text { física }\end{array}$ & IMC \\
\hline \multirow{6}{*}{$\begin{array}{l}\text { Rho de } \\
\text { Spearman }\end{array}$} & \multirow{3}{*}{$\begin{array}{l}\text { Actividad } \\
\text { física }\end{array}$} & $\begin{array}{l}\text { Coeficiente de } \\
\text { correlación }\end{array}$ & 1,000 &,$- 204^{* *}$ \\
\hline & & Sig. (bilateral) & . & ,002 \\
\hline & & $\mathrm{N}$ & 234 & 234 \\
\hline & \multirow{3}{*}{ IMC } & $\begin{array}{l}\text { Coeficiente de } \\
\text { correlación }\end{array}$ &,$- 204^{* *}$ & 1,000 \\
\hline & & Sig. (bilateral) & ,002 & . \\
\hline & & $\mathrm{N}$ & 234 & 234 \\
\hline
\end{tabular}

**. La correlación es significativa en el nivel 0,01 (bilateral).

En la correlación Rho de Spearman entre las variables actividad física y el IMC es negativa baja de $-0,204$ con un nivel de significancia de $\mathrm{P}=0,002$ $<0,01$; por lo que se determina, una correlación inversamente proporcional entre las variables actividad física y el estrés. Ello significa que a medida que aumenta la actividad física disminuye el IMC.

\section{Conclusiones}

El contexto actual producto de la COVID-19 y el SARS-Cov-2 están ocasionando en las personas incremento de la inactividad física, disminución del gasto energético, consumo excesivo de alimentos hipercalóricos, estrés, y ansiedades generadas por el aislamiento, que impactan en el índice de masa corporal (IMC). Los docentes universitarios presentan mayores porcentajes de nivel de actividad física baja, en contraste con el nivel de actividad alta donde los varones muestran mayor práctica frente a las mujeres.

Respecto al estrés se encontró que los docentes tienen mayor estrés por presión laboral, depresión y ansiedad producto de la emergencia sanitaria, y la asunción de las diferentes responsabilidades académicas de los docentes. En el índice de masa corporal los varones muestran porcentajes más altos de sobrepeso (obesidad grado I) y existe una prevalencia mayor hacia la obesidad grado II y III en comparación con las mujeres donde evidencian una tendencia mayor hacia el sobrepeso (obesidad grado I), obesidad grado II, III y IV.

Se requiere implementar medidas integrales con enfoque interdisciplinario centrados en el estilo de vida saludable de los docentes con dieta y la combinación de actividad física y ejercicio físico sistemáticos acompañados de terapias psicológicas, con la finalidad de optimizar el ejercicio profesional de los docentes universitarios.

\section{Agradecimientos}

A la Universidad Nacional del Altiplano, en especial a los docentes que nos permitieron realizar dicha investigación en plena emergencia sanitaria.

El financiamiento de la presente investigación ha sido a cargo de la Universidad Nacional del Altiplano, a través del Fondo Especial de Desarrollo Universitario (FEDU).

\section{Conflicto de intereses}

Los autores declaran no tener ningún conflicto de interés

\section{Referencias bibliográficas}

Acosta, M., Parra, L., Burbano, C., Aguilera, M.Á., y Pozos Radillo, B. E. (2019). Estrés laboral, burnout, salud mental y su relación con violencia psicológica en docentes universitarios. Salud Uninorte, 35(3), 328342. http://www.scielo.org.co/scielo.php? script $=\mathrm{sci}$ arttext\&pid=S 0120 55522019000300328

Aljuhani, O., Alkahtani, S., Alhussain, M., Smith, L., \& Habib, S. S. (2020). Associations of physical activity and sedentary time with metabolic syndrome in saudi adult males. Risk Management and Healthcare Policy, 13, 1839-1847. 10.2147/RMHP.S267575

Alvitesí, C. (2019). Estrés docente y factores psicosociales en docentes de Latinoamérica, Norteamérica y Europa TT - Teacher Stress and Psychosocial Factors in Teachers from Latin America, North America and Europe. Journal of Indo - European Studies, 47(3/4), 141-159. https://bbibliograficas.ucc.edu.co/ docview/2331807229? accountid $=44394$

Aparicio, V. A., Soriano-Maldonado, A., Buitrago, F., Félix-Redondo, F. J., \& Fernández-Bergés, D. (2016). The Role of Sex and Domestic Physical Activity on the Metabolically Healthy and Unhealthy Obesity. The HERMEX Study. Revista Española de Cardiología, 69(10), 983-986. 10.1016/i.recesp.2016.04.041

Arias, W.L., Huamani, J.C., y Ceballos, K.D. (2019). Síndrome de Burnout en profesores 
de escuela y universidad: un análisis psicométrico y comparativo en la ciudad de Arequipa. Propósitos y Representaciones, 7(3), 72-110. http://www.scielo.org.pe/pdf/ pyr/v7n3/a04v7n3.pdf

Arrieta, F., y Pedro-Botet, J. (2020). Reconocer la obesidad como enfermedad: todo un reto. Revista Clínica Española, xx, 1-3. 10.1016/j. rce.2020.08.003

Bedoya, E.A., Vega, N.E., Severiche, C.A., y Meza, M. J. (2017). Síndrome de Quemado (Burnout) en Docentes Universitarios: El Caso de un Centro de Estudios del Caribe Colombiano TT - Burnout Syndrome in University Teachers: the Case of a Study Center in the Colombian Caribbean. Formación Universitaria, 10(6), 51-58. http://www.scielo.cl/scielo.php?script=sci arttext\&pid=S0718-50062017000600006\&la ng=es\%0Ahttp://www.scielo.cl/pdf/ formuniv/v10n6/art06.pdf

Brais, A., Leirós, R., \& García Soidán, J.L. (2021). Are adults an influential role model in children's physical activity habits? An observational study of the Spanish population. Retos, 2041(39), 306-311. 10.47197/retos. v0i39.78528

Brazo, J., Mielke, G.I., Olivares, P.R., Jahnecka, L., \& Crochemore M. Silva, I. (2018). Descriptive Epidemiology of Uruguayan Adults' Leisure Time Physical Activity. International Journal of Environmental Research and Public Health, 15(7), 1387. 10.3390/ijerph15071387 Burgos, C., Henríquez, C., Ramírez, R., Mahecha, S., y Cerda Kohler, H. (2017). ¿Puede el ejercicio físico per se disminuir el peso corporal en sujetos con sobrepeso/obesidad? Revista Médica de Chile, 145(6), 765-774. 10.4067/s0034-98872017000600765

Córdova, J.Á. (2016). La obesidad: la verdadera pandemia del siglo XXI. Cirugía y Cirujanos, 84(5), 351-355. 10.1016/j.circir.2016.08.001

Di Blasio, A., Di Donato, F., y González-Castro, C. (2017). Cuestionario Internacional de Actividad Física para uso con jóvenes y adultos de mediana edad (15-69) (IPAQ).

Dong, X., Li, Y., Zhang, L., Liu, X., Tu, R., Wang, Y., Li, R., Li, L., Hou, J., Mao, Z., Huo, W., \& Wang, C. (2021). Independent and interactive effect of sitting time and physical activity on prevalence of hyperuricemia: the Henan Rural Cohort Study. Arthritis Research and Therapy, 23(1), 1-10. 10.1186/s13075-020$\underline{02385-8}$
Enriquez, L.A., Cervantes, N., Candia, R., y Flores, L.A. (2021). Capacidades físicas y su relación con la actividad física y composición corporal en adultos. Retos, 41, 674-683. 10.47197/ retos.v41i0.83067

Escobar, C., y Divisón, J. A. (2016). Actividad física: ¿es la intensidad importante? Semergen, 42(3), 190-191. 10.1016/j. semerg.2015.07.001

Fernández-Lázaro, D., González-Bernal, J. J., Sánchez-Serrano, N., Navascués, L. J., Del Río, A. A., \& Mielgo-Ayuso, J. (2020). Physical exercise as a multimodal tool for COVID-19: Could it be used as a preventive strategy? International Journal of Environmental Research and Public Health, 17(22), 1-13. 10.3390/ijerph17228496

Feter, N., Caputo, E. L., Smith, E. C., Doring, I. R., Cassuriaga, J., Leite, J. S., Reichert, F. F., da Silva, M. C., Coombes, J. S., \& Rombaldi, A. J. (2021). Association between physical activity and subjective memory decline triggered by the COVID-19 pandemic: Findings from the PAMPA cohort. Preventive Medicine, 145(December 2020), 106415. 10.1016/j.ypmed.2020.106415

Flores, A., Pino, Y.M., y Villamar, M.O. (2019). Actividad física y su relación con el sobrepeso y obesidad en docentes de la Universidad Nacional del Altiplano Puno 2017. Sportis. Scientific Journal of School Sport, Physical Education and Psychomotricity, 6(1), 61-79. 10.17979/sportis.2020.6.1.3448

Font, C., Mur, E., Bort, J., Gomes, M., y Milà, R. (2020). Effects of mild intensity physical activity on the physical condition of older adults: A systematic review. Revista Española de Geriatria y Gerontología, 55(2), 98-106. 10.1016/j.regg.2019.10.007

García, A.J. y Froment, F. (2018). Beneficios de la actividad física sobre la autoestima y la calidad de vida de personas mayores. Retos. Nuevas Tendencias En Educación Física, Deporte y Recreación, 33, 3-9.

Gómez, J.M., y Martínez-Marcos, M. (2018). Sobrepeso y obesidad: eficacia de las intervenciones en adultos. Enfermeria Clínica, 28(1), 65-74. 10.1016/j.enfcli.2017.06.005

Hernández-Sampieri, R., Fernández-Collado, C. y Baptista-Lucio, P. (2006). Metodología de la investigación (M.-H. Interamericana (ed.); Cuarta Edi).

Hernández, A., Monguí, K., y Rojas, Y. (2018). Descripción de la composición corporal, 
fuerza muscular y actividad física en pacientes con insuficiencia renal crónica en hemodiálisis en una unidad renal en Bogotá, Colombia. Revista Andaluza de Medicina Del Deporte, 11(2), 52-56. 10.1016/j.ramd.2016.09.005

Hsiao, C., Shiang, W., \& Peng, W. (2020). The experience of menopausal women participating in weight management program: A pilot study. Taiwanese Journal of Obstetrics and Gynecology, 59(5), 686-690. 10.1016/j. tjog.2020.07.011

Iglesias, S. L., Azzara, S. H., Gonzalez, D., Ibar, C., Jamardo, J., Berg, G. A., Bargiela, M. del M., \& Fabre, B. (2018). Stress management program for students, teachers and nonteachers of the Faculty of Pharmacy and Biochemistry of the University of Buenos Aires. Ansiedad y Estres, 24(2-3), 105-111. 10.1016/j.anyes.2018.07.003

Liévano, M., Ospina, J., López, M., Liévano, M., y Villamizar, L. (2015). Prevalencia de sedentarismo y factores asociados en estudiantes y docentes de postgrado de una facultad de medicina de Bogotá, Colombia. La Sociedad Madrileña de Medicina Familia y Comunitaria, 2(17), 7-14.

Limón,M.R.yOrtega,M.C.(2011).Envejecimiento activo y mejora de la calidad de vida en adultos mayores. Revista de Psicología de La Educación. 6, 6.

López, P.J., Madrona-Marcos, F., PaniselloRoyo, J., Carbayo-Herencia, J. A., Rosich, N., Tarraga-Marcos, L., Castell, E., y Alins, J. (2020). Evaluación de un programa de intervención motivacional de actividad física en el tratamiento de la obesidad y el sobrepeso. Hipertensión y Riesgo Vascular, 37(1), 11-16. 10.1016/j.hipert.2019.05.003

Martínez, N., Santaella, E., y Rodríguez, A.M. (2020). Beneficios de la actividad física para la promoción de un envejecimiento activo en personas mayores. Revisión bibliográfica (Benefits of physical activity for the promotion of active aging in elderly. Bibliographic review). Retos, 2041(39), 829834. 10.47197/retos.v0i39.74537

Mejia, C.R., Failoc, V.E., Cárdenas, M. M. y Rodriguez, J.F. (2020). Factores sociolaborales asociados a la variación del IMC y diámetro de cintura en trabajadores de 15 ciudades peruanas. Rev. Asoc. Esp. Espec. Med. Trab, 29, 236-244.

Mesurado, B., y Laudadío, J. (2019). Experiencia profesional, capital psicológico yengagement.
Su relación con el burnout en docentes universitarios. 7(3), 12-26.

Monterrey, P., y Porrata, C. (2001). Procedimiento gráfico para la evaluación del estado nutricional de los adultos según el índice de masa corporal. Rev Cubana Aliment Nutr, 15(1), 62-67. https://www.researchgate.net/ publication/262068529\%0 A PROCEDIMIENTO

Morales, J., Matta, H., Fuentes-Rivera, J., Pérez, R., Suárez, C., Alvines, D., \& Carcausto, W. (2018). Excess weight and cardiometabolic risk among faculty members at a private university of Lima: Opportunity to build healthy environments. Educación Médica, 19, 256-262. 10.1016/i.edumed.2017.08.003

Naranjo, J., Álvarez, J., Blasco, C., \& Gaztañaga, T. (2021). Obesity and physical exercise in adults. Archivos de Medicina Del Deporte, 37(5), 326-337. 10.18176/ ARCHMEDDEPORTE.0007

Nepal, A., Karki, U., Poudel, L., Rajbhandari, B., \& Wagle, S. (2020). Prevalence of overweight among adult women of a metropolitan. Journal of the Nepal Medical Association, 58(227), 474-479. 10.31729/jnma.5190

Oblitas, L., Anicama, J., Bayona, L., Bazán, C., \& Ferrel, F. (2020). Impacto del mindfulness en el estrés en trabajadores de una universidad de Lima sur. Salud Uninorte, 35(2), 238-249.

OMS. (2010). Recomendaciones mundiales sobre actividad física para la salud. Ginebra Suiza.

OMS. (2020). Estrategia mundial sobre régimen alimentario, actividad física y salud. Ginebra Suiza.

Ortiz, R., Torres, M., Siguencia, W., SigüenzaCobos, N., Salazar, J., Añez, R., Rojas, J., y Bermúdez, V. (2017). Influencia de la actividad física y el consumo calórico sobre la adiposidad visceral en adultos de la ciudad de Cuenca, Ecuador. Revista Argentina de Endocrinología y Metabolismo, 54(4), 160 168. 10.1016/j.raem.2017.07.007

Ortiz, B., De León, L.G., Esparza, J., Carrasco, C.E. y Candia, R. (2019). Ejercicio moderado y consumo de alimentos de alto y bajo índice glucémico en mujeres sedentarias. Atención Primaria, 51(6), 327-332. 10.1016/j. aprim.2018.02.008

Palacios, M.E., y Montes de Oca, V. (2017). Condiciones de Trabajo y Estrés en Académicos Universitarios. Ciencia \& Trabajo, 19(58), 49-53. 10.4067/s0718$\underline{24492017000100049}$ 
Ramírez, R., González, K., García, S., y Agredo, R.A.(2012). Diferencias por sexo de la relación entre ejercicio de intensidad vigorosa vs. intensidad moderada y marcadores de riesgo de soprepeso/obesidad en adultos saludables. Endocrinología y Nutrición, 59(8), 491-495. 10.1016/j.endonu.2012.06.011

Rondanelli, I. R. y Rondanelli, S. R. (2014). Estilo de vida y enfermedad cardiovascular en el hombre. Revista Médica Clínica Las Condes, 25(1), 69-77.10.1016/s0716-8640(14)70013$\underline{6}$

Royo-Bordonada, M. Á., Rodríguez-Artalejo, F., Bes-Rastrollo, M., Fernández-Escobar, C., González, C. A., Rivas, F., MartínezGonzález, M. Á., Quiles, J., BuenoCavanillas, A., Navarrete-Muñoz, E. M., Navarro, C., López-García, E., Romaguera, D., Morales Suárez-Varela, M., \& Vioque, J. (2019). Políticas alimentarias para prevenir la obesidad y las principales enfermedades no transmisibles en España: querer es poder. Gaceta Sanitaria, 33(6), 584-592. 10.1016/j. gaceta.2019.05.009

Russo, M.J., Kañevsky, A., Leis, A., Iturry, M., Roncoroni, M., Serrano, C., Cristalli, D., Ure, J., y Zuin, D. (2020). Papel de la actividad física en la prevención de deterioro cognitivo y demencia en adultos mayores: una revisión sistemática. Neurología Argentina, 12(2), 124-137.

Salas, C., Peterman, F., Adela, M., María, A., Díaz, X., Garrido, A., Poblete-Valderrama, F., y Celis-Morales, C. (2018). Poco es mejor que nada: Practicar actividad física de manera regular podría reducir a la mitad el riesgo de desarrollar diabetes mellitus. Revista Médica Clínica Las Condes, 29(1), 98-100. 10.1016/j. rmclc.2017.11.009

Santos, M.R.F., Da Silva, M.E., \& Belmonte, B.D.R. (2021). COVID-19: Emergency remote teaching and university professors' mental health. Revista Brasileira de Saude Materno Infantil, 21, S245-S251. 10.1590/1806-9304202100s100013

Simón, R.M., Sánchez, A.J., Suárez, W., \& González, J.A. (2020). Effect of a physical exercise program on physical fitness and visceral fat in people with obesity. Retos, 2041(39), 723-730. 10.47197/retos. v0i39.78997

Stewart, A., Marfell-Jones, M., Olds, T., y H. de R. (2011). Protocolo internacional para la valoración antropométrica. Sociedad Internacional para el avance de la cineantropometria.

Tacca, D.R., y Tacca, A.L. (2019). Factores de riesgos psicosociales y estrés percibido en docentes universitarios. Propósitos y Representaciones, 7(3), 323. 10.20511/ pyr2019.v7n3.304

Tejada, J., Tejada, J., y Nuviala, A. (2016). Actividad física y salud. https://elibro.net/es/ ereader/unapuno-biblioteca/44792?page $=115$

Tojal, L., Alonso-Gómez, A., Alberich, S., Wärnberg, J., Sorto, C., Portillo, M.P., Schröder, H., Salas-Salvadó, J., \& Arós, F. (2020). Association between maximal oxygen consumption and physical activity and sedentary lifestyle in metabolic syndrome. Usefulness of questionnaires. Revista Española de Cardiologia, 73(2), 145-152. 10.1016/j.recesp.2018.08.014

Villalobos, F., Vinuesa, A., Pedret, R., Reche, A., Domínguez, E., y Arija, V. (2019). Efecto de un programa de actividad física sobre la autoestima en sujetos con enfermedades crónicas. Ensayo de intervención comunitaria "Pas a Pas." Atención Primaria, 51(4), 236244. 10.1016/j.aprim.2017.11.011 